\title{
Dealing with Futile Treatments Based on the Humanistic Approach
}

\author{
Parham Pooladgar ${ }^{1}$ and Shabnam Bazmi (10) ${ }^{2, *}$ \\ ${ }^{1}$ School of Medicine, Shahid Beheshti University of Medical Sciences, Tehran, Iran \\ ${ }^{2}$ Medical Ethics Department, School of Traditional Medicine, Shahid Beheshti University of Medical Sciences, Tehran, Iran \\ Corresponding author: Medical Ethics Department, School of Traditional Medicine, Shahid Beheshti University of Medical Sciences, Tehran, Iran. Email: \\ sh_bazmi2003@yahoo.com
}

Received 2020 November 24; Revised 2020 December 15; Accepted 2020 December 19.

\begin{abstract}
Medical futility is one of the most common controversial topics in medicine, medical ethics, and philosophy of medicine. Every day, doctors are dealing with patients who are in a condition that must have a decision about requested futile treatment with their own beliefs, opinions, and different demands. This is an important issue that must be taught during teaching courses. Therefore, it is good to provide general policies for teaching how to make the best decision, establishing better communication between doctors and patients, and maintaining their Autonomy. On the other hand, with the interference and expansion of humanistic and holistic attitudes in the treatment of patients, it must be considered that at all treatment levels, especially decisions related to end-of-life, it is better to involve this point of view in our policies. In the present article, we tried to give a general conclusion of general policy and present standards for a humanistic policy by analyzing various countries' policies and expressing their bugs.
\end{abstract}

Keywords: Medical Futility, Humanistic Medicine, Philosophy of Medicine, Ethics, Teaching

\section{Context}

The concept of medical futility and the reason for its existence are related to the essence of medicine. Therefore, this subject is as old as medicine itself (1). Hippocrates addressed this concept in his writings. It is also encountered in Plato's works $(2,3)$. However, the definition of medical futility is controversial; it can be considered as the inappropriate use of medical interventions, such as diagnostic, preventive, and therapeutic interventions, or treatments that do not have any advantages for the patient $(4,5)$. While some studies define futile treatments merely as treatments that do not achieve their intended aims, they can also be characterized as interventions that are inordinately unsuccessful or have no definite benefits in the treatment process (6). The concept of medical futility is applicable in various settings but is mostly applied to end-of-life situations. Therefore, most examples regarding medical futility concern these situations $(7,8)$.

Most discussions about the definition and usage of medical futility involve value and belief conflicts between patients, or their families, and physicians. Discussions regarding medical futility may also involve differences between patients and their physicians concerning the purpose and method of treatment (7).

There are several definitions of medical futility:
- Physiological futility: Futility can be defined in terms of physiological objectives. Thus, a treatment that has no physiological and medical effect is considered futile (7, 9 ). In some instances, providing a physiologically useless treatment by physicians and healthcare providers may be considered a medical mistake even if it is made following the wish of the patient or his family $(7,10)$.

- Normative futility: This is a treatment that has valuable results for the patient and his family, but the physician, while knowing that the treatment may have some physiological benefits (for example, increases the patient's life expectancy), considers it useless in general, based on the treatment goals (11).

- Quantitative futility: When physicians conclude (through either personal experience, experiences shared by colleagues, or consideration of published empirical data) that a certain medical treatment has been useless in the last 100 cases, they should regard that treatment as futile (12).

- Qualitative futility: It refers to instances in which an intervention fails to lead to an acceptable quality of life for the patient. When treatment is judged to be qualitatively futile, the argument is made that although the treatment may succeed in achieving an effect, the effect is not worth achieving from the patient's perspective (10). Qualitative futility is also described as disproportional futility (7). 
Physicians frequently encounter patients for whom certain treatments are futile, even if patients and their families might believe otherwise. Therefore, physicians need to know how to deal with such situations, especially when the patient and his family insist on receiving futile treatments (13). At the same time, it must be borne in mind that medical and financial resources and medical sciences have their limitations (5). Also, according to studies, it seems that teaching the principles of medical ethics and concepts such as medical futility to physicians and treatment staff can lead to greater readiness and better decision-making in complex challenges. Also, it can reduce disputes and discussions between the treatment team, the patient, and his family (14).

Different countries display differences in terms of culture, customs, and religious matters and beliefs that can influence specific decisions regarding medical futility (5). Therefore, some countries, even some hospitals, are considering developing policies to help them work systematically towards resolving conflicts on medical futility decisions (5).

Different countries follow different policies about medical futility, but in this study, we attempt to divide these policies into two general categories based on the review of the sources:

- General/authority policy: In this category, general policies are designed for dealing with the issue of medical futility, describing the authorities and decision-making processes regarding the issue. These general policies do not address details and exceptions in medical futility cases but are more concerned with recommendations, such as the policy proposed in Kansas City.

- Details/order policy: A kind of policy that specifies what should be considered as medical futility and what should not. This type of policy addresses details of medical futility. It describes the tasks and decision-makers in detail. The policy proposed in Hong Kong can be considered in this category.

Next, we address the humanistic perspective and concepts regarding medical futility. We will draw upon these considerations in discussing the humanistic aspects of policies regarding medical futility.

\subsection{The Humanistic Perspective on Medicine}

Conventional medicine (biomedicine) was besieged by numerous difficulties because it focused solely on the physiological problems of patients and did not pay attention to their wishes, beliefs, and other dimensions of their being, such as mental and spiritual dimensions, and their perceived suffering and illness.

This stance leads to challenges between the physician, patient, and the patient's family, termed "quality of care crisis" by Marcum (15). The humanistic approach to medicine tried to resolve these challenges and introduced holistic medicine. In this approach, a patient, in addition to their physiological problems, is a set of thoughts, desires, and values that affect them and their treatment processes. Conventional and traditional medicine considers a person a machine composed of several organs and systems. Break-down of one of these organs leads to disease, and the patient's problem will be treated by treating and repairing that organ. This perspective originated from Newton's mechanistic monism philosophy. But, from the humanistic point of view, a person, in addition to the body, has a mental dimension, and these two elements affect each other. The humanistic view sees a person as a two-dimensional or multidimensional being, so during illness and treatment, it is necessary to pay attention to their desires and values. The humanistic view posits that the source of suffering during an illness is the feeling by the person who is separated from the others because of the illness and cannot control his body anymore. Therefore, the physician should try to exercise empathy and compassion with the patient and take his beliefs into account in the treatment process. As a result, the physician should make individual decisions regarding patients even if they have the same physiological problems (15).

\subsection{Pathos: A Bridge between Conventional Medicine and Hu- manistic Medicine}

Pathos is the state of being that is the basis of all possible wise and loving actions. As propounded by Aristotle, pathos is one of the basic elements of rhetoric and public speaking. It is primarily related to passion and the effect of speech on the audience. Even if it is transient and imperfect, pathos can take ascendancy over the other two principles of rhetoric, which are ethos (lecturer's credibility) and logos (rationality, logic, and accuracy of speech) $(15,16)$.

Application of the art or science of medicine and common approaches in the practice of medicine, such as evidence-based medicine (EBM) and patient care management (PCM), can result in a fundamental problem in the communication between the physician and the patient, called the quality of care crisis, as mentioned earlier. To solve this issue, medicine should be combined with pathos. Therefore, changing the discourse from a biomedical framework to a humanistic one is not enough. The physician should transcend from ethos and logos to pathos and passion. The practitioner should try to communicate with the patient and understand their pain and help him know that his suffering is felt. Then, the physician should try to motivate the patient to continue with the treatment and overcome the pain. Pathos tries to solve the quality care crisis through two approaches: (1) pathos 
tries to turn the logos, technics, facts, objective science, and subjective information into wisdom; (2) pathos can turn the biomedical physician's ethos, or even the humanistic physician's empathy, into love between the patient and the physician and solve the quality of care crisis in this way (15).

\section{Statement of Problem}

Given the widening acceptance of the humanistic view in medicine and greater communication between the patient and the physician and their joint participation in making decisions, the question arises whether policies regarding medical futility are humanistic? Or, in other words, what are the features of a humanistic policy on medical futility? Moreover, according to the introduction, these concepts (medical futility, humanistic medicine, and pathos) and medical futility decision-making policies must be taught to undergraduate medical students and medical staff that encounter medical futility cases, including ICU physicians, to solve the challenges better. Therefore, what features should have these education curricula to be more effective?

Here, we address policies presented in various countries, critically examine their strengths and weaknesses in terms of philosophy and ethics, and classify them into the two main types of policies mentioned earlier in the study in a bid to establish the characteristics of a humanistic policy.

Following the recommended guidelines of the Kansas City Medical Ethics Committee, seven groups of specialists in the field of medical futility deliberated questions in this field and tried to answer these questions to reach specific guidelines. In this study, patients' feelings and physicians' responsibilities regarding the topic of medical futility are adequately addressed, and cultural, religious, and belief differences are considered. Efforts were also made to fully recognize patient autonomy and respect their family's decisions. In summary, the proposed guidelines are as follows:

-The first stage: Investigation of the benefits and harms of continuing the treatment and discussing the futility of treatment.

- The second stage: Patients' effective communication with their families and explanation of the situation to them.

- The third stage: In cases where the patient and his family reject the physician's recommendations, the ethics committee will examine the issue and try to resolve the conflict between the patient and the physician. In effect, the committee tries to guide both sides.
- The fourth stage: If the patient or his family does not insist on continuing the treatment, it is terminated, and the patient enters palliative care. However, if the patient or his family insists on continuing the futile treatment, they should be well. Also, the patient can change his present doctor, and his previous doctor can help too.

- The fifth stage: If the two sides do not agree with the third and fourth stages, a specialist, called the mediator, intervenes and gives his opinion after analyzing the situation. The specialist should have adequate training and qualifications to evaluate all aspects of the situation.

- The sixth stage: In the event of a persisting disagreement, the patient or his family is given the option to either choose another physician to continue the treatment or discontinue the treatment and start palliative care $(13,17)$.

In Belgium guidelines, as in the U.S., the patient's refusal to undergo treatment is generally respected, and his request for a futile treatment is not accepted (7). In this country, euthanasia is legal and is considered as part of palliative care (18). In final decisions regarding medical futility, the hospital ethics committee and the regional ethical legislation assembly are involved. The physician can delegate the treatment to a colleague and leave the treatment team. If the patient is not conscious, and the physician sees no physiological benefits in the treatment, it can be stopped in consultation with the hospital ethics committee, even if the family is opposed to stopping the futile treatment $(7,19)$. Such a stance stems from the Belgian people's view on good life and good death (18).

A study examined the end-of-life maintenance treatment in critically ill patients in Hong Kong in connection to controversial medical futility issues. The article first addresses the difference between euthanasia and abandonment of futile treatment and notes that, in Hong Kong, active euthanasia is illegal and considered immoral. As in other articles, two common definitions of medical futility are presented, namely, physiological and normative definitions. The article further argues that there is no difference between futile treatments withholding and withdrawing futile treatment in the opinion of the clinical ethics committee, and both are similar in terms of their legal and moral burdens. Then, the article discusses the person sanctioned to make decisions regarding futile treatments: (1) the request of a mentally competent and mature person should be respected if he does not wish to continue the treatment; (2) when the patient is incapable of making decisions, a guardian or family member takes over that responsibility; (3) for a person who does not have a family or a guardian, the hospital ethics committee makes the decision.

The article emphasizes that the treatment team is not obliged to perform a treatment with no physiological ef- 
fects. In general, the levels of authority are as follows: First, the patient and the physician together work towards making a decision. If they cannot agree on one, the case goes to the ethics committee. The committee decides whether the treatment should be stopped. Alternatively, the patient can be referred to another physician that continues the treatment (20).

South Korea is a country that, in contrast to many other countries, including Iran, accepts voluntary euthanasia. At the same time, in this country, unlike in Western countries, the individual's autonomy has a less significant role than the decision of the family and guardian in medical matters (21). With an increase in life expectancy in South Korea, the number of age-associated chronic diseases requiring intensive care grew, and subsequently, in 1997, the following code of medical ethics was adopted: "The physician should try to reduce the mental and physical suffering of terminally ill patients and help them to accept death."

In 2002, the Korean Academy of Medical Sciences developed the following guideline regarding terminal patients: (1) patient autonomy must be respected; (2) the decision to end or continue treatment should be made based on the patient's quality of life and the benefits; (3) the decision about the futility of treatment should be made cautiously, and after the physician, their colleagues, and the hospital ethics committee reviewed it (7).

In the United States, courts generally agree that the family's and patient's opinion about the continuation of futile treatment is important and should be taken into account. Attempts were made in Texas and Virginia so that physicians could end futile treatment legally. Texas laws were enacted in 1999, and Virginia passed similar laws in the following years. The Texas law, numbered 166.052, enumerates under what circumstances a physician can end the treatment unilaterally.

If the physician concludes that the treatment will be futile, the patient and his caregiver should be notified 48 hours in advance of the commencement of a committee set up to decide so that they can attend the committee meeting. If the committee agrees that the treatment is futile, the physician and the committee will give 10 days to the patient to find another medical facility to continue the treatment. After 10 days, they can legally discontinue the treatment unless the court sets an additional 10-day reprieve. In Virginia, 14 days are given to the patient without the confirmation of the committee (7).

Unlike the United States (Texas and Virginia), there are no rules in Japan on medical futility. There is no accepted definition of medical futility in Japan, but there are guidelines regarding end-of-life. The proposed guideline about end-of-life care in emergency treatments, introduced in 2007 , addresses conditions where the treatment is not use- ful but will cause the patient to suffer and lose dignity, and neither the patient nor his family considers continuing it desirable. This guideline concerns four kinds of situations: (1) there is irreversible damage to the brain function; (2) there is dependence on life support devices and ventilators due to irreversible and fatal failure of body organs in the absence of alternative treatments; (3) the patient will die in the next few days, even if treatment continues; (4) the patient has reached the final stage of malignancy or irreversible disease despite rigorous and aggressive treatment.

This guideline stipulates that life-prolonging therapies can be discontinued, but the patient and his family and healthcare providers should be consulted. If the patient or his family wants treatment to be continued, it will be done. The Japanese Ministry of Health announced in 2007 that, in addition to the parties mentioned above, an ethics committee is needed. In 2008, the medical association of Japan stated that the quality of life should be accorded more importance, and continuing the treatment for life at the cost of losing it is not right. Therefore, the need to end treatment and discontinue it should be considered more closely (7).

In Iran currently, there are no laws regarding medical futility and no proper guidelines for making decisions in this regard in the country. However, it is felt that a special protocol is needed to decide on cases involving medical futility, and the medical community and legislators should propose it, and these protocols must be taught in medical education courses. The Charter of Patients' Rights of Iran was published in 2009. It includes five topics, one of them dealing with end-of-life issues.

The relevant clause in the charter first defines appropriate treatment. According to the charter, every individual has the right to receive appropriate treatment consistent with the sanctity of the human being, customs of the society, and their religious beliefs. In cases where death is unavoidable, appropriate treatment should be provided to reduce patient suffering (7).

Two treatment situations can be interpreted as inactively helping the critically ill patient to die. First, the use of analgesics to reduce the patients' physical pain without the intention to kill, an intervention that can shorten the patient's life. Unless it is intended to kill the patient, attempting to alleviate pain or other serious physical symptoms of the patient is not considered a crime and is permitted under Islamic law. The second situation involves the discontinuation of treatment based on the joint decision of the physician, the patient, his family, and other parties involved in the treatment to forgo treatment aimed at delaying death. In Iran and most other Muslim countries, it is believed that the human body is owned by God, so no one, 
even the patient and his family, has the right to sanction death (euthanasia).

\section{Results}

We found that the step-by-step decision-making framework (general, authority policy) rather than an array of sub-frameworks (details, order policy) serves one of our general goals, namely respecting the autonomy and values of patients, their representatives, and their families. Moreover, this framework respects the autonomy of the physician and clinical team and helps resolve the quality of care crisis (13).

The guidelines proposed in Kansas City are fully consistent with our initial assumption of the need for a stepby-step decision-making framework. But, the following critiques should also be considered:

- How much money and time should be spent on training people for the mediation position? Is there a better solution?

- Can a person in the role of a mediator fulfill their expected obligations? Can a person understand all the physical and mental aspects of futility questions in all patients and make the right decisions?

- Are conflicts of interest more likely in decisionmaking by individuals rather than by groups?

- Can the treatment be stopped if the patient, for any reason, refuses to refer to another medical facility or cannot afford to do so, or if the center where the patient is being treated is the only place that provides the medical service in question?

Finally, it seems that placing a person at the top of the decision-making pyramid both reduces the accuracy of decision-making in various situations and increases the likelihood of the interference of personal interests in the decision-making process. Besides, it is difficult for one individual to have a sense of compassion and pathos and complete understanding for all patients.

A critique of the recommended guidelines in Belgium is that they focus on the social and autonomous freedoms of the patient, especially in the field of euthanasia, without considering the possibility that decisions and autonomy of the patient may compromise the autonomy of his family and those around him. For example, if the ill father of a family decides to refuse treatment or opts for euthanasia, he will be well within his rights according to the law, but would his wife and children agree with this decision? Would the autonomy of those around the patient not be compromised if the patient's autonomy was respected and his wish was granted despite his wife's opposition? It is conceivable that the harm inflicted on those around the patient by narrowly focusing on the patient's autonomy might be greater than the harm that comes to the patient by denying his wish.

Regarding the Hong Kong guideline, in the first situation, where the patient is his own decision-maker, the request of a mentally competent, mature, and alert person is respected and implemented. However, it should be ensured that the person is aware of all aspects of his decision and its consequences. In the second situation, if the patient cannot make decisions for any reason, his family members or a guardian decide for him. A critique of this arrangement is that the decisions of the family members or guardians may be against the patient's possible wishes. In the third situation, if the patient cannot make a decision and does not have a family or a guardian, the hospital ethics committee decides for him. A potential critique of this arrangement is the possibility of the committee being oblivious to the patient's values and preferences.

The following further critiques can be offered regarding the article about maintenance therapy in the end-oflife guideline:

- In Hong Kong guidelines, active euthanasia is specifically addressed, but there is no clear discussion of passive euthanasia. The guidelines recommend that withdrawing and withholding futile therapy should not be equated with passive euthanasia because of the semantic connotations of the word "euthanasia", but this argument does not seem to be valid. On the contrary, as Yu Kam Por's study shows, these two concepts are equivalent in some aspects, essentially when referring to a single entity, and have the same result. However, they look at the same treatment and intervention issues from two different angles, namely, physician's and patient's perspectives (22).

- The humanistic approach strives to establish pathos and compassion between the physician and the patient and acknowledge the patient's beliefs and culture, but unfortunately, this topic is not adequately addressed, and this is the most significant shortcoming of the paper.

- There is no higher decision-making authority above the level of the ethics committee, and this situation can result in problems. The arbitrating authority is not clear if there is an objection to the committee's verdict. Moreover, the higher institution has a kind of supervisory role over the lower authorities, which is not defined here.

The same critique that was brought forth regarding the Belgian law on euthanasia, applies to the Korean guidelines, namely, the issue of patient autonomy versus the autonomy of relatives and those around the patient. Therefore, this point should be considered.

The consideration of the quality of life and the benefit of the patient and the emphasis that decisions should be beneficial to the patient are among the highlights of this policy. These considerations are in line with the general 
policy and the hierarchy considered in the introduction of this study. The most salient critiques regarding the American rules and guidelines are summarized below:

- The first point is that the physician and the committee can stop the treatment if they find it futile. Here, it is not clear whether this authority applies to normatively futile treatments, physiological futile treatments, or both.

- According to the Virginian law, the physician can pronounce a treatment futile at any time and for any reason, and there is no higher committee to monitor and review the case. As a result, this law can be considered a kind of paternalism because the physician can impose his will on the patient, and the law does not restrain him.

- The question arises: Is it morally acceptable to deprive the patient of his wishes due to a conflict of values or lack of resources, or to force him to either find another place of treatment or forego the treatment? This situation appears to be incompatible with the humanistic view.

A common approach to the issue of end-of-life and medical futility in Iran is that if the control of pain and physical symptoms in the patients entails premature death, the physician has no right to attempt to curb the symptoms, even if it is the patient's will. In many jurisprudences, the termination of treatment is explicitly forbidden, and the patient's wish to do so is denied. In Islam, no human being has the authority over his death, and any attempt in this direction is considered sinful interference in the work of God and is forbidden. As a result, it is inconsistent with humanistic views and the notion of pathos in some ways, and in some cases, the patient's autonomy is limited, and his values and desires are taken into account.

\section{Conclusions}

After reviewing the literature, we conclude that a codified policy, like the Kansas City guideline, is required to solve this problem and improve decision-making. The features of such a policy, informed by the humanistic approach, are discussed below.

From a humanistic point of view, the patient's values and beliefs must be considered, and the patient's autonomy should be cherished. Therefore, an individually tailored approach should be employed for each patient. The following points should be addressed so that decisionmaking is regulated; at the same time, patient autonomy is maintained, and putting them under duress is avoided:

- Based on the considerations addressed in the introduction, an authorization policy is generally in line with the humanistic view. Therefore, fixed decision-making powers should not be invested in patients or healthcare providers. For example, if a patient suffering from a certain illness requests that a specific action be taken regard- ing his treatment, the humanistic point of view demands that the decision be made after examining the patient's desires and values. As a result, the policy only offers general rules for decision-making while maintaining the patient's and physician's (health provider) autonomy.

- A codified policy, even of the authority or general type, can reduce the level of compassion and pathos between the physician and the patient because it can lead to a speedy referral of disputed issues to higher authorities. To illustrate this point, we take a situation where the physician and patient do not agree on the futility of the treatment. If the policy stipulates that in such cases, the matter should be referred to the ethics committee, the physician might refrain from further discussions and refer the matter to higher authorities forthwith to avoid the challenge with the patient and his family, particularly if the physician has previously had undesirable and bad experiences in similar situations. This course of action would diminish the physician's compassion and empathy with the patient, an issue that has special significance in the humanistic perspective. Therefore, the physician and the patient must be in prolonged contact with each other to maintain compassion among them and solve the above problems. The physician and patient should attend all meetings throughout the decision-making process. Moreover, the higher authorities should have some supervisory role and examine all aspects of the process, and if there is a violation at any stage, they should deal with it.

- Another important issue is that the placement of certain fixed people as decision-makers with higher authority, such as the ethics committee, and their inflexibility may result in ignoring the cultural, religious, and value beliefs of patients. Thus, pathos will not be established, and this is a potential shortcoming of the policy. The following example illustrates this point. Suppose that a Buddhist from another country with a different culture is hospitalized in countries like Iran, and a situation involving futile treatment develops. There is a national policy in this regard; several structures are in place at the hospital, such as the ethics committee, which comprises mainly Iranian, Mus$\mathrm{lim}$, and Shiite members. Can these experts look at the issue from this person's point of view and comment accordingly? Would this person not feel that his values have not been adequately considered and a decision has been imposed on him as a result of geographical and temporal determinism? This scenario is even valid for cases from the same country because there are differences in beliefs and values in different regions of a country. To function in a truly humanistic way, the policy needs to be flexible in designating decision-making authorities and strive to relate to the patient's thoughts, culture, religion, and native language as much as possible. Thus, compassion and pathos 
are established between the health care providers, and patients and obtaining results is facilitated.

After reviewing the literature and critically examining current policies in different countries, we conclude that each of them has its own shortcomings. However, an authority policy, under the humanistic perspective, meets the desired criteria.

The medical ethics contents can be categorized into knowledge (values, concepts, and medical ethics principle) and attitudes (compassion, honesty, pathos, and empathy). Traditional medical ethics education programs mostly emphasize knowledge in medical ethics courses and pay less attention to attitudes for a variety of reasons, such as lack of time, lack of facilities, and emphasis on the traditional approach to memorizing content rather than applied medical ethics education and Problem-based Learning (PBL) approaches $(23,24)$. But, we need attitudes, humanistic approaches, and critical thinking skills in medical ethics education curricula to train good doctors who can encounter well with most ethical challenges like medical futility. Thus, to have a better understanding and appropriate education medical futility concept, new educational strategies are needed, which include: (1) preparing a suitable guideline for solving medical futility challenges in Iran; (2) educating this guideline to medical students at different levels; (3) evaluating the impact of education on medical staff and how they encounter with the issue of medical futility after education; (4) educating new entities and concepts like concepts of medical philosophy (humanistic perspective, patient-centered medicine, etc.), critical thinking skills, physician-patient communication issues (compassion, pathos, and empathy), and even general ethics (23) in addition to medical ethics; (5) application of role playing and simulation in education with the participation of students (23); (6) application of films, lectures (23), and PBL in medical ethic courses; (7) educating ethics in a practical way and practical training on the patient's bedside; and (8) educating the subject of medical futility by people who have studied in this field or have similar experiences (25).

More research is needed on the subject of medical futility to construct a humanistic policy in collaboration with physicians, patients, medical ethics committees, medical philosophers, health managers, and policymakers, a policy that can maintain the physician and patient autonomy and help them resolve their disagreements more effectively.

At the same time, it should be noted that there is a fundamental lack of a local policy on medical futility based on religious and cultural considerations and using the principles of humanism and medical ethics in Iran. We need to have an acceptable policy and teach these subjects to our graduated and undergraduate physicians. It is hoped that experts in medical sciences, medical ethics, medical philosophy, medical education, legislators, and religious jurists can work together to fill this gap.

\section{Footnotes}

Authors' Contribution: Sh. Bazmi, design and final revision; P. Pooladgar, design and writing.

Conflict of Interests: None declared.

Funding/Support: None declared.

\section{References}

1. Schneiderman LJ. Defining medical futility and improving medical care. J Bioeth Inq. 2011;8(2):123-31. doi: 10.1007/s11673-011-9293-3. [PubMed: 21765643]. [PubMed Central: PMC3106156].

2. Whitmer M, Hurst S, Prins M, Shepard K, McVey D. Medical futility: A paradigm as old as Hippocrates. Dimens Crit Care Nurs. 2009;28(2):6771. doi: 10.1097/DCC.ob013e318195d43f. [PubMed: 19225315].

3. Scannell K, Henry SC. Medical Futility. Perm J. 2002;6(1):52-4. [PubMed: 30313013]. [PubMed Central: PMC6220620].

4. Aramesh K. [Medical futility]. J Med Ethics Hist Med. 2008;1(4):47-52. Persian.

5. Bagheri A. Medical futility: Is a policy needed? J Clinic Res Bioeth. 2014;5(5). e102. doi: 10.4172/2155-9627.1000e102.

6. Danis M, Truog R, Devita M, Dagi F, Englehardt T, Grenvik A, et al. Consensus statement of the Society of Critical Care Medicine's Ethics Committee regarding futile and other possibly inadvisable treatments. Crit Care Med. 1997;25(5):887-91. doi: 10.1097/00003246199705000-00028. [PubMed: 9187612].

7. Bagheri A. Medical Futility: A Cross-National Study. London, UK: Imperial College Press; 2013. doi: 10.1142/p881.

8. Jox RJ, Schaider A, Marckmann G, Borasio GD. Medical futility at the end of life: The perspectives of intensive care and palliative care clinicians. J Med Ethics. 2012;38(9):540-5. doi: 10.1136/medethics-2011100479. [PubMed: 22562948].

9. Saeedi Tehrani S, Madani M. [Bioethical principles and medical futility].J Med Ethics Hist Med. 2015;7(6):1-14. Persian.

10. Clark PA. Medical futility: Legal and ethical analysis. Virtual Mentor.2007;9(5):375-83. doi:10.1001/virtualmentor.2007.9.5.msoc1-0705. [PubMed: 23217998].

11. Jacobs BB, Taylor C. Medical futility in the natural attitude. ANS Adv Nurs Sci. 2005;28(4):288-305. doi: 10.1097/00012272-20051000000002. [PubMed: 16292016].

12. Aghabarary M, Nayeri ND. Medical futility and its challenges: A review study.J Med Ethics Hist Med. 2016;9. [PubMed: 28050241]. [PubMed Central: PMC5203684].

13. Brannigan MC. On medical futility: Considerations and guidelines. Mo Med. 2006;103(2):113-7. [PubMed: 16703706].

14. Sulmasy DP, Geller G, Levine DM, Faden RR. A randomized trial of ethics education for medical house officers. $J$ Med Ethics. 1993;19(3):157-63. doi: 10.1136/jme.19.3.157. [PubMed: 8230148]. [PubMed Central: PMC1376284]

15. Marcum JA. An introductory philosophy of medicine: Humanizing modern medicine. Berlin, Germany: Springer Science \& Business Media; 2008.

16. Dan B. Medical rhetoric and rhetoric medicine. Dev Med Child Neurol. 2014;56(10):916. doi: 10.1111/dmcn.12577. [PubMed: 25208967].

17. Center for Practical Bioethics. Recommended policy guidelines regarding medical futility. Kansas City, Missouri, USA: Kansas City Area Ethics Committee Consortium, Center for Practical Bioethics; 2006. 
18. Bernheim JL, Deschepper R, Distelmans W, Mullie A, Bilsen J, Deliens L. Development of palliative care and legalisation of euthanasia: Antagonism or synergy? BMJ. 2008;336(7649):864-7. doi: 10.1136/bmj.39497.397257.AD. [PubMed: 18420693]. [PubMed Central: PMC2323065].

19. Carlet J, Thijs LG, Antonelli M, Cassell J, Cox P, Hill N, et al. Challenges in end-of-life care in the ICU. Statement of the 5th international consensus conference in critical care: Brussels, Belgium, April 2003. Intensive Care Med. 2004;30(5):770-84. doi: 10.1007/s00134-004-2241-5. [PubMed: 15098087].

20. Working Group on Modular Review of HA Guidelines on LifeSustaining Treatment. HA guidelines on life-sustaining treatment in the terminally ill. 2. Hong Kong: Hospital Authority: Patient Safety \& Risk Management Department; 2015.
21. Heo DS. Life-sustaining medical treatment for terminal patients in Korea. J Korean Med Sci. 2013;28(1):1-3. doi: 10.3346/jkms.2013.28.1.1 [PubMed: 23341705]. [PubMed Central: PMC3546086].

22. Kam Por Y. Terminating Futile Medical Treatment and Passive Euthanasia: Is there a Difference? Eubios Journal of Asian and International Bioethics. 2002;12(4):137-8.

23. Giubilini A, Milnes S, Savulescu J. The medical ethics curriculum in medical schools: Present and future. J Clin Ethics. 2016;27(2):129-45 [PubMed: 27333063].

24. Schwartzstein RM. Getting the right medical students-nature versus nurture. N Engl J Med. 2015;372(17):1586-7. doi: 10.1056/NEJMp1501440. [PubMed: 25901425].

25. Stirrat GM. Teaching and learning medical ethics and law in UK medical schools. ClinicalEthics. 2010;5(3):156-8. doi:10.1258/ce.2010.010029. 